\title{
X-ray Thomson Scattering in Warm Dense Matter without the Chihara Decomposition
}

\author{
A. D. Baczewski, ${ }^{1,}$ L. Shulenburger, ${ }^{2}$ M. P. Desjarlais, ${ }^{2}$ S. B. Hansen, ${ }^{2}$ and R. J. Magyar ${ }^{1}$ \\ ${ }^{1}$ Center for Computing Research, Sandia National Laboratories, Albuquerque, New Mexico 87185, USA \\ ${ }^{2}$ Pulsed Power Sciences Center, Sandia National Laboratories, Albuquerque, New Mexico 87185, USA
}

(Received 17 December 2015; published 18 March 2016)

\begin{abstract}
$\mathrm{X}$-ray Thomson scattering is an important experimental technique used to measure the temperature, ionization state, structure, and density of warm dense matter (WDM). The fundamental property probed in these experiments is the electronic dynamic structure factor. In most models, this is decomposed into three terms [J. Chihara, J. Phys. F 17, 295 (1987)] representing the response of tightly bound, loosely bound, and free electrons. Accompanying this decomposition is the classification of electrons as either bound or free, which is useful for gapped and cold systems but becomes increasingly questionable as temperatures and pressures increase into the WDM regime. In this work we provide unambiguous first principles calculations of the dynamic structure factor of warm dense beryllium, independent of the Chihara form, by treating bound and free states under a single formalism. The computational approach is real-time finite-temperature time-dependent density functional theory (TDDFT) being applied here for the first time to WDM. We compare results from TDDFT to Chihara-based calculations for experimentally relevant conditions in shock-compressed beryllium.
\end{abstract}

DOI: 10.1103/PhysRevLett.116.115004

Warm dense matter (WDM) arises in many contexts ranging from planetary science [1-3] to the implosion stage of inertial confinement fusion [4-6]. While there are no sharp pressure, temperature, and density boundaries for the WDM regime, it is generally viewed as an intermediate state between a condensed phase and an ideal plasma where Fermi degeneracy is present, and the Coulomb coupling and thermal energy are comparable in magnitude [7].

Experimental characterization of warm dense matter is challenging due to the difficulty of producing uniform samples at extreme conditions and developing diagnostic techniques that can provide accurate and independent measurements of these conditions for transient samples opaque to optical photons. X-ray Thomson scattering (XRTS) $[7,8]$, one such diagnostic technique, exploits the scattering of hard coherent $\mathrm{x}$ rays to directly probe the system's dynamic structure factor (DSF). Through the fluctuation-dissipation theorem, the DSF is related to the system's density-density response, and, consequently, XRTS provides direct insight into electron dynamics.

XRTS experiments have been performed on a variety of materials including beryllium [9,10], lithium [11], carbon [12], CH shells [13], and aluminum [14,15]. With recent improvements in source brightness [15] producing increasingly high resolution and high signal-to-noise data, the full DSF is expected to become routinely available. In anticipation of these advances, it is critical and timely to

Published by the American Physical Society under the terms of the Creative Commons Attribution 3.0 License. Further distribution of this work must maintain attribution to the author(s) and the published article's title, journal citation, and DOI. examine the theoretical constructs underpinning the interpretation of these experiments.

The most common model of XRTS experiments relies on an additive form of the DSF due to Chihara [16,17]:

$$
\begin{aligned}
S(\mathbf{q}, \omega)= & \left|f_{I}(\mathbf{q})+\rho(\mathbf{q})\right|^{2} S_{i i}(\mathbf{q}, \omega)+Z_{f} S_{e e}(\mathbf{q}, \omega) \\
& +S_{b f}(\mathbf{q}, \omega) .
\end{aligned}
$$

The DSF varies with momentum and energy transfers ( $\mathbf{q}$ and $\omega$ ) and is partitioned into 3 features that can be interpreted in terms of $\mathrm{x}$-ray scattering processes. These include scattering from electrons bound to and adiabatically following ions $\left[\left|f_{I}(\mathbf{q})+\rho(\mathbf{q})\right|^{2} S_{i i}(\mathbf{q}, \omega)\right]$, from $Z_{f}$ free electrons per ion $\left[Z_{f} S_{e e}(\mathbf{q}, \omega)\right]$, and from bound electrons that are photoionized $\left[S_{b f}(\mathbf{q}, \omega)\right]$. While successfully applied to many systems, this model relies on numerous approximations and assumptions. Most critically, the electrons are separated into bound and free populations, a distinction that is often ambiguous in the WDM regime. Each term in Eq. (1) is subject to different models potentially leading to underconstrained fits to experimental data [18]. The ionic feature typically relies upon a decomposition into a product of an ion-ion structure factor, $S_{i i}(\mathbf{q}, \omega)$, and an average atomic form factor, $f_{I}(\mathbf{q})+\rho(\mathbf{q})$, with the first term describing the unscreened bound electrons and the latter the screening cloud, which must be treated carefully [19]. However, recent work has focused on moving past this decomposition of the elastic peak [20].

In this work, we transcend the Chihara decomposition by explicitly simulating the real-time dynamics of warm dense matter using a finite temperature form of time-dependent density functional theory (TDDFT) [21,22] and the 
projector augmented-wave (PAW) formalism [23,24]. In PAW, the all-electron Kohn-Sham orbitals (and their associated density) can be accessed via an explicit linear transformation on the smoother pseudo orbitals. By performing calculations with none of the core states frozen, we avoid making any distinctions between bound and free electrons, and treat all electrons similarly.

We work with a real-time implementation of TDDFT in the Vienna $a b$ initio simulation package (VASP) [24-26] (see Supplemental Material [27]) that provides a number of attractive features. Physically, higher-order response phenomena and Ehrenfest molecular dynamics are accessible in this framework. Computationally, the orthogonalization bottleneck that limits standard DFT approaches is removed, as it is only explicitly required for the calculation of the initial state of the Kohn-Sham orbitals. This leads to excellent strong scaling $[38,39]$, and we have observed near-perfect scaling up to 65536 cores in our implementation.

We next outline the details of our TDDFT calculations, noting that we use Hartree atomic units $\left(m_{e}=e^{2}=\hbar=\right.$ $\left.1 / 4 \pi \epsilon_{0}=1\right)$ unless otherwise indicated. Time-dependent quantities evaluated at $t=0$ are indicated by the addition of a subscript 0 and the absence of a temporal argument. Fourier transformed quantities are indicated by a diacritic tilde. All calculations are spin unpolarized.

The equation of motion in real-time TDDFT is the timedependent Kohn-Sham (TDKS) equation,

$$
i \frac{\partial}{\partial t} \phi_{n, \mathbf{k}}(\mathbf{r}, t)=\left(-\frac{\nabla^{2}}{2}+v_{S}[\rho](\mathbf{r}, t)\right) \phi_{n, \mathbf{k}}(\mathbf{r}, t),
$$

in which $v_{S}[\rho](\mathbf{r}, t)=v_{\text {ext }}(\mathbf{r}, t)+v_{H}[\rho](\mathbf{r}, t)+v_{x c}[\rho](\mathbf{r}, t)$. The orbitals, indexed by band and Bloch wave number, are such that their weighted sum produces the time-dependent density, $\rho(\mathbf{r}, t)$. The external potential, $v_{\text {ext }}$ includes contributions due to the Coulomb field of the bare nuclei as well as a model of the x-ray probe, $v_{\text {probe }}(\mathbf{r}, t)$, which is quiescent until $t=0 . v_{H}$, and $v_{x c}$ are the Hartree and exchange-correlation potentials, with accurate and efficiently computable approximations to the latter being a central theoretical concern of TDDFT.

The initial conditions for the $\left\{\phi_{n, \mathbf{k}}(\mathbf{r}, t)\right\}$ from Eq. (2) are the self-consistent solution to a Kohn-Sham Mermin DFT calculation [40] at electron temperature $T_{e}$ in a supercell of volume $\Omega_{s c}$. The initial density is then

$$
\rho_{0}(\mathbf{r})=\sum_{n, \mathbf{k}} f_{n, \mathbf{k}}\left(T_{e}\right)\left|\phi_{n, \mathbf{k}, 0}\left[T_{e}\right](\mathbf{r})\right|^{2},
$$

where $f_{n, \mathbf{k}}$ is a composite weight consisting of the measure of the specific Bloch orbital and the Mermin weight encoding temperature dependence according to a FermiDirac distribution. It is important to note the implicit dependence of these initial orbitals on the equilibrium electron temperature, $T_{e}$. Evolving these orbitals under Eq. (2), the time-dependent density becomes

$$
\rho(\mathbf{r}, t)=\sum_{n, \mathbf{k}} f_{n, \mathbf{k}}\left(T_{e}\right)\left|\phi_{n, \mathbf{k}}(\mathbf{r}, t)\right|^{2} .
$$

The weights are not time evolved, as we do not expect them to change in the linear response regime [41]. For stronger perturbations, additional formalism might be required for the weights. That the Mermin formalism is sensible within TDDFT in the linear response regime is supported by recent foundational work [42].

The action of a probe potential, $v_{\text {probe }}(\mathbf{r}, t)$, turned on at $t=0$ leads to a change in the time-dependent density. The linear density-density response function, $\chi_{\rho \rho}\left(\mathbf{r}, \mathbf{r}^{\prime}, t\right)$, encodes the relationship between these two quantities

$$
\delta \rho(\mathbf{r}, t)=\int_{0}^{\infty} d \tau \int_{\Omega_{s c}} d \mathbf{r}^{\prime} \chi_{\rho \rho}\left(\mathbf{r}, \mathbf{r}^{\prime}, \tau\right) \delta v_{\mathrm{ext}}\left(\mathbf{r}^{\prime}, t-\tau\right),
$$

where we use the notation $\delta f(\mathbf{r}, t)=f(\mathbf{r}, t)-f_{0}(\mathbf{r})$ for $\delta \rho(\mathbf{r}, t)$ and $\delta v_{\text {ext }}(\mathbf{r}, t)$. If we fix ionic positions at time $t=0$, which evolve slowly on the relevant attosecond time scales, then $\delta v_{\text {ext }}(\mathbf{r}, t)=v_{\text {probe }}(\mathbf{r}, t)$ and we can construct a probe potential that can be used to extract the DSF, similar to Sakko et al. [43]. The real-time density response to such a probe potential is shown in Fig. 1. (a)

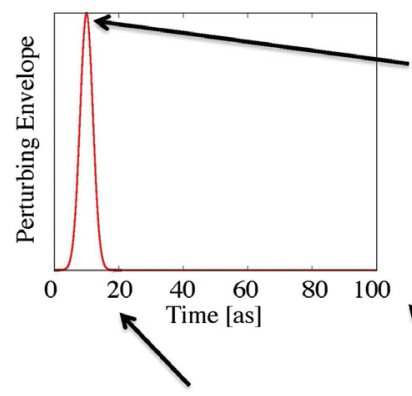

(c)

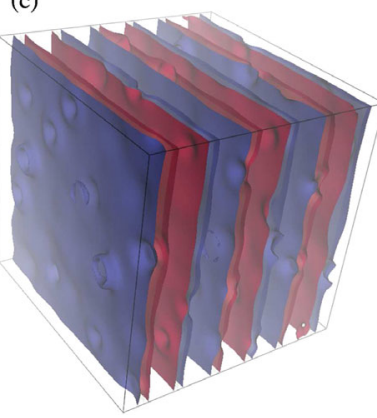

(b)

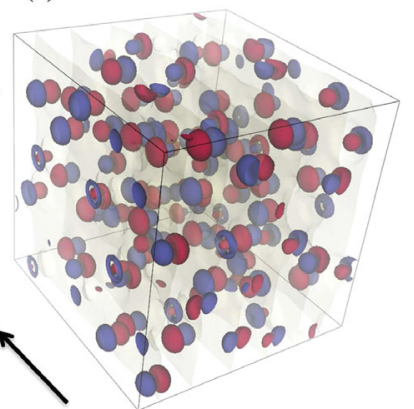

(d)

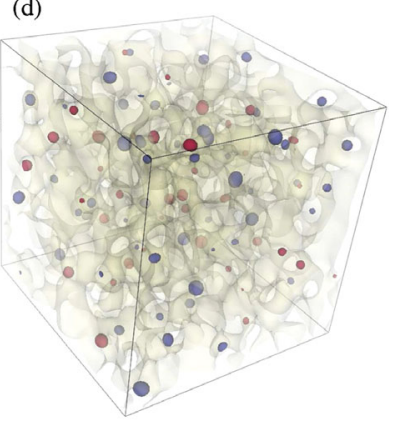

FIG. 1. The density response of warm dense beryllium (density $5.5 \mathrm{~g} / \mathrm{cm}^{3}$ and $T_{e}=13 \mathrm{eV}$, movie in the Supplemental Material [27]) due to (a) a perturbing potential with the illustrated envelope observed at times coinciding with (b) the peak of the perturbation, (c) the peak of the density response, and (d) the plasmons continuing to ring around the system after the perturbation has ceased. Red (blue) isosurfaces bound volumes of charge accumulation (depletion) and yellow isosurfaces indicate the nodal surface. The amplitude of the perturbation is within the linear response regime, a discussion of which is in the Supplemental Material [27]. 
In principle, any sufficiently weak analytic probe potential will allow us to extract the response function. For convenience, we choose $v_{\text {probe }}(\mathbf{r}, t)=v_{0} e^{i \mathbf{q} \cdot \mathbf{r}} f(t)$, where $f(t)$ is a Gaussian envelope and $v_{0}$ is related to the probe intensity. The Fourier transformed response function, $\tilde{\chi}_{\rho \rho}(\mathbf{q},-\mathbf{q}, \omega)=\delta \tilde{\rho}(\mathbf{q}, \omega) / v_{0} \tilde{f}(\omega)$, is then related to the DSF through the fluctuation-dissipation theorem

$$
S(\mathbf{q}, \omega)=-\frac{1}{\pi} \frac{\operatorname{Im}\left[\chi_{\rho \rho}(\mathbf{q},-\mathbf{q}, \omega)\right]}{1-e^{-\omega / k_{b} T_{e}}} .
$$

We are careful to note that Fourier transforms are normalized such that $\delta \tilde{\rho}(\mathbf{q}, \omega)$ has units of inverse frequency.

As a proof of principle, we report calculations of the DSF for $3 \mathrm{x}$-compressed beryllium consistent with the conditions reported in Ref. [10]. We consider the same momentum transfers as in Ref. [44] to study a range of excitations spanning the collective regime to the beginning of noncollective regime. For convenience of presentation, each $\mathbf{q}$ value is mapped onto an XRTS scattering angle, $\theta$, relative to the $2 \AA$ probe wavelength in Ref. [10] (see Supplemental Material [27] for more information). Our results come from averaging the response of electronic densities generated from several static uncorrelated ionic configurations sampled from thermally equilibrated DFT-MD calculations. These calculations were performed on 32 and 64 atom supercells with a four electron beryllium PAW potential within the local density approximation (LDA), with electrons and ions thermostatted at $T=13 \mathrm{eV}$, and $\mathbf{k}$ point sampling at $\left(\frac{1}{4}, \frac{1}{4}, \frac{1}{4}\right)$, analogous to the Baldereschi mean-value point for cubic supercells. For these conditions a plane wave cutoff of $1400 \mathrm{eV}$ was required to converge the pressure to within 1\%, and 576(32 atom)/ 1152 (64 atom) Kohn-Sham orbitals were needed to represent the thermal occupation $\left[f_{n, \mathbf{k}}\left(T_{e}\right) \geq 10^{-5}\right]$.

Each sample configuration is used to seed a TDDFT calculation of the DSF, utilizing the same cutoff and number of orbitals. The initial Mermin electronic state is recomputed using a denser $\mathbf{k}$-point sampling on a $3 \times 4 \times 4$ (32 atom) or $2 \times 2 \times 2$ (64 atom) Monkhorst-Pack grid. To assess the effect of the frozen core approximation (FCA), we consider electronic initial conditions and dynamics generated using both two and four electron beryllium PAW potentials. Results of our calculations are illustrated in Figs. 2 and 3. Details of the averaging procedure used to generate results, and information concerning the satisfaction of sum rules, can be found in the Supplemental Material [27].

Figure 2 directly compares the DSF computed with and without the FCA. While the PAW method still includes the proper all-electron density in aggregate, only the orbitals tied to the two outermost valence electrons are included in the time-evolved response within the FCA. This effectively eliminates the dynamics of the core states from the density response and the high energy shoulder above $80 \mathrm{eV}$ in the

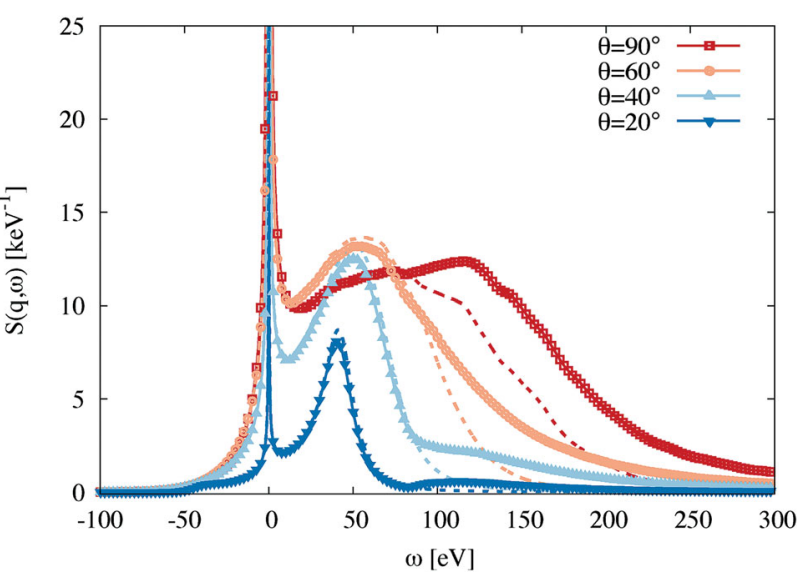

FIG. 2. The DSF of warm dense beryllium (density $5.5 \mathrm{~g} / \mathrm{cm}^{3}$ and $T_{e}=13 \mathrm{eV}$ ) at the scattering angles $(\theta)$ considered in Ref. [44] with (dashed lines) and without (marked lines) the frozen core approximation. All TDDFT calculations utilize the adiabatic LDA.

DSF is removed. For the temperatures and densities being considered, this is roughly equivalent to partitioning the inner two and outer two electrons into bound and free groups in the Chihara picture, such that the two-electron response corresponds to $S_{e e}(\mathbf{q}, \omega)$. However, there are important distinctions to keep in mind. First, in the four-electron calculation, all electrons are being treated identically, whereas it is typical to treat $S_{e e}(\mathbf{q}, \omega)$ and $S_{b f}(\mathbf{q}, \omega)$ using different levels of theory and without self-consistency in the Chihara framework. Second, even in the two-electron calculation, the response of the outer two electrons is still aware of the two frozen core states tied to each atom through their screening of the nuclear potential. Finally, these calculations are based upon explicit simulations of the real-time electron dynamics of a bulk supercell of warm dense beryllium rather than a phenomenological model of the response based upon a jellium plus average-atom picture.

Based upon our observation that the two-electron response roughly corresponds to $S_{e e}(\mathbf{q}, \omega)$, we can also extract a quantity akin to $S_{b f}(\mathbf{q}, \omega)$ by differencing the four-electron and two-electron DSFs. The effective $S_{e e}(\mathbf{q}, \omega)$ and $S_{b f}(\mathbf{q}, \omega)$ computed within TDDFT are illustrated in Fig. 3. Here we compare our TDDFT calculations to calculations done using state-of-the-art models for $S_{e e}(\mathbf{q}, \omega)$ and $S_{b f}(\mathbf{q}, \omega)$. The former is treated with an RPA-level model dielectric function with lifetime effects taken from four-electron DFT-MD calculations of the optical conductivity, the Mermin approximation ab initio collision frequencies (MA-AICF) method in Ref. [44]. The latter is calculated with the formalism developed in [45] and a quantum mechanical average-atom ion-sphere model with Slater exchange. As we are interested in studying energies relevant to the electronic response, we ignore $S_{i i}(\mathbf{q}, \omega)$, though it is necessarily present in the Chihara-independent TDDFT calculation. 

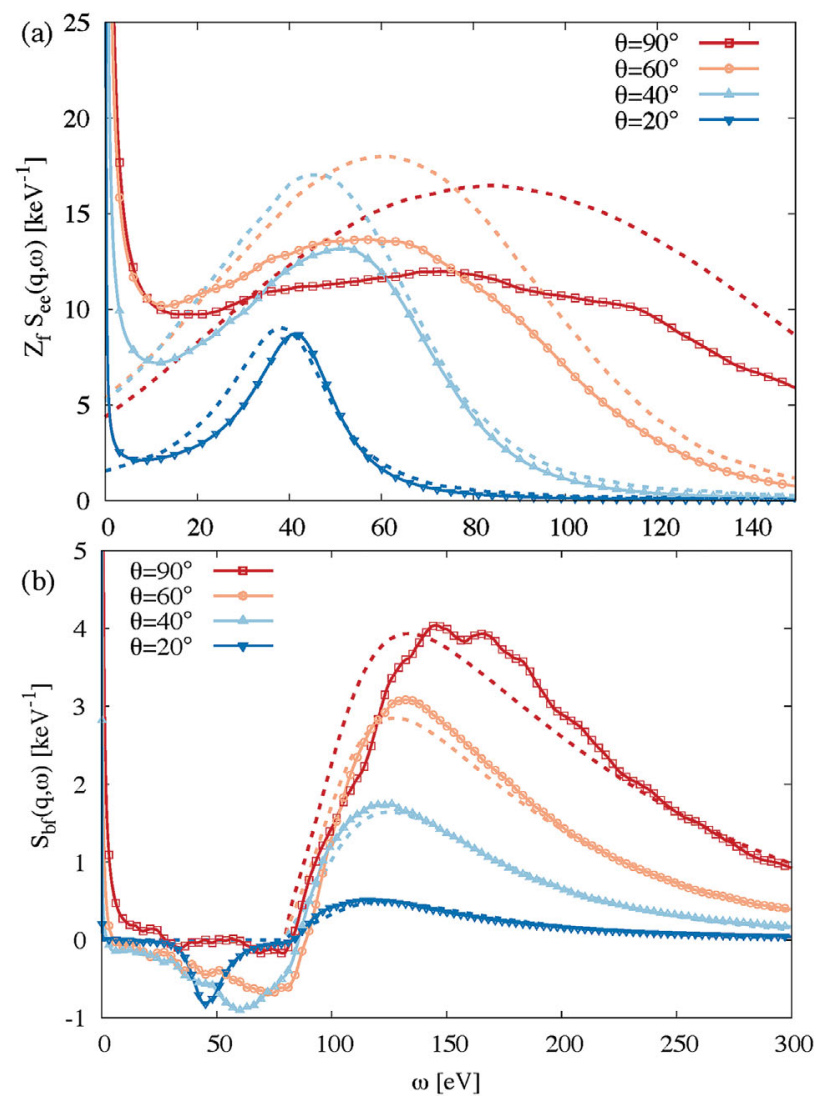

FIG. 3. Comparison of the DSF of warm dense beryllium computed using TDDFT (marked lines) and individual terms in a Chihara decomposed theory (dashed lines). (a) Illustrates the twoelectron TDDFT response compared to the MA-AICF method [44] for $Z_{f} S_{e e}(\mathbf{q}, \omega)$. (b) Illustrates the difference of the four- and two-electron TDDFT responses compared to an average atom treatment [45] of $S_{b f}(\mathbf{q}, \omega)$.

Examining the dispersion of the primary plasmon peak in Fig. 3(a), we see that TDDFT predicts a slight $(\sim 5 \mathrm{eV})$ blueshift relative to the MA-AICF calculation $\theta=20^{\circ}$ and $40^{\circ}$, whereas it predicts a stronger $(\sim 10 \mathrm{eV})$ redshift relative to MA-AICF at $\theta=60^{\circ}$ and $90^{\circ}$. We attribute this shift to exchange or correlation and band structure effects, not present in the MA-AICF dielectric function. Previously, comparisons of inelastic $\mathrm{x}$-ray scattering spectra to RPA and LDA in cold free electron metals $(\mathrm{Na}$ and $\mathrm{Al})$ indicate a similar trend in which both LDA and experiment are redshifted relative to RPA [46]. However, these calculations also indicate that the addition of lifetime effects to the LDA are necessary to totally reconcile theory and experiment. While nonadiabatic exchange correlation kernels are available for energy domain linear response TDDFT, no such time-domain exchange correlation potentials are currently available for real-time TDDFT. As warm dense matter requires a large number of thermally occupied states such that energy domain TDDFT may become computationally prohibitive, warm dense matter may provide compelling motivation for the development and testing of these functionals.
Considering the bound-free shoulder in Fig. 3(b), we see that TDDFT is generally in good agreement with the average-atom model treatment of $S_{b f}(\mathbf{q}, \omega)$ with some minor differences. Such average atom models agree well with real-space Green's function methods for cold solid beryllium [47]. We observe a trend opposite the free-free feature, in which there is a redshift of the TDDFT result at small angles, and a blueshift at large angles. We expect that LDA will do an increasingly poor job of describing the Compton scattering limit at large $\theta$ due to its wellestablished self-interaction error. Applying a functional with some fraction of Fock exchange should remedy this behavior and will be the subject of future investigations. Further, the TDDFT bound-free feature computed by differencing the two- and four-electron DSFs has a small negative dip below the $80 \mathrm{eV}$ onset of the core feature. It is difficult to determine whether this is due to core-polarization suppressing the response of the valence electrons in the four-electron calculation, or potentially an artifact of the different pseudization procedures used to generate the two PAW potentials used for this comparison. This motivates further investigations of the PAW formalism applied to both real-time TDDFT and specifically, warm dense matter in which thermal effects will start to blur the line between core and valence electrons.

We presented a method for the direct calculation of the DSF for warm dense matter, independent of the Chihara decomposition, by applying real-time TDDFT to configurations drawn from thermal Mermin DFT-MD calculations. Comparison of our results with state-of-the-art models applied within the Chihara picture illustrates some subtle differences between the two, though it generally supports the use of the Chihara formalism as an inexpensive alternative to the very detailed and computationally intensive TDDFT calculations. We anticipate that TDDFT may provide a powerful discriminating tool for arbitrating disagreements between these more phenomenological theories and experiment, especially as experimental data become more highly resolved. Our framework enables future explorations of systems in which the partition between bound and free electrons is more ambiguous. It also provides a platform for studying the impact of recent foundational developments in DFT at nonzero temperature $[42,48-53]$.

Calculations were completed on Chama and Skybridge at Sandia National Laboratories and Sequoia at Lawrence Livermore National Laboratory. We are grateful for discussions with Stephen Bond, Kieron Burke, Hardy Gross, Ryan Hatcher, Neepa Maitra, Thomas Mattsson, Normand Modine, Jonathan Moussa, Kai-Uwe Plagemann, Aurora Pribram-Jones, Kenneth Rudinger, Travis Sjostrom, and Sam Trickey. A. D. B, L. S., M. P. D., and R. J. M. were supported by Sandia's Laboratory Directed Research and Development (LDRD) Project No. 165731. S. B. H. was supported by the U.S. Department of Energy, Office of Science Early Career Research Program, Office of Fusion 
Energy Sciences. Sandia National Laboratories is a multiprogram laboratory managed and operated by Sandia Corporation, a wholly owned subsidiary of Lockheed Martin Corporation, for the U.S. Department of Energy's National Nuclear Security Administration under Contract No. DE-AC04-94AL85000.

*adbacze@sandia.gov

[1] T. Guillot, Science 286, 72 (1999).

[2] J. J. Fortney and N. Nettelmann, Space Sci. Rev. 152, 423 (2010).

[3] R. Kraus, S. Stewart, A. Seifter, and A. Obst, Earth Planet. Sci. Lett. 289, 162 (2010).

[4] M. K. Matzen et al., Phys. Plasmas 12, 055503 (2005).

[5] R. P. Drake, Phys. Plasmas 16, 055501 (2009).

[6] S. X. Hu, L. A. Collins, T. R. Boehly, J. D. Kress, V. N. Goncharov, and S. Skupsky, Phys. Rev. E 89, 043105 (2014).

[7] S. Glenzer and R. Redmer, Rev. Mod. Phys. 81, 1625 (2009).

[8] G. Gregori, S. H. Glenzer, W. Rozmus, R. W. Lee, and O. L. Landen, Phys. Rev. E 67, 026412 (2003).

[9] S. H. Glenzer, O. L. Landen, P. Neumayer, R. W. Lee, K. Widmann, S. W. Pollaine, R. J. Wallace, G. Gregori, A. Höll, T. Bornath, R. Thiele, V. Schwarz, W.-D. Kraeft, and R. Redmer, Phys. Rev. Lett. 98, 065002 (2007).

[10] H. J. Lee, P. Neumayer, J. Castor, T. Döppner, R. W. Falcone, C. Fortmann, B. A. Hammel, A. L. Kritcher, O. L. Landen, R. W. Lee, D. D. Meyerhofer, D. H. Munro, R. Redmer, S. P. Regan, S. Weber, and S. H. Glenzer, Phys. Rev. Lett. 102, 115001 (2009).

[11] E. G. Saiz, G. Gregori, D. O. Gericke, J. Vorberger, B. Barbrel, R. Clarke, R. R. Freeman, S. Glenzer, F. Khattak, M. Koenig et al., Nat. Phys. 4, 940 (2008).

[12] C. R. D. Brown, D. O. Gericke, M. Cammarata, B. Cho, T. Döppner, K. Engelhorn, E. Förster, C. Fortmann, D. Fritz, E. Galtier et al., Sci. Rep. 4, 5214 (2014).

[13] L. Fletcher, A. Kritcher, A. Pak, T. Ma, T. Döppner, C. Fortmann, L. Divol, O. Jones, O. Landen, H. Scott et al., Phys. Rev. Lett. 112, 145004 (2014).

[14] T. Ma, T. Döppner, R. Falcone, L. Fletcher, C. Fortmann, D. O. Gericke, O. Landen, H. Lee, A. Pak, J. Vorberger et al., Phys. Rev. Lett. 110, 065001 (2013).

[15] L. Fletcher, H. Lee, T. Döppner, E. Galtier, B. Nagler, P. Heimann, C. Fortmann, S. LePape, T. Ma, M. Millot et al., Nat. Photonics 9, 274 (2015).

[16] J. Chihara, J. Phys. F 17, 295 (1987).

[17] J. Chihara, J. Phys. Condens. Matter 12, 231 (2000).

[18] A. N. Souza, D. J. Perkins, C. E. Starrett, D. Saumon, and S. B. Hansen, Phys. Rev. E 89, 023108 (2014).

[19] K.-U. Plagemann, H. R. Rüter, T. Bornath, M. Shihab, M. P. Desjarlais, C. Fortmann, S. H. Glenzer, and R. Redmer, Phys. Rev. E 92, 013103 (2015).

[20] J. Vorberger and D. O. Gericke, Phys. Rev. E 91, 033112 (2015).

[21] C. A. Ullrich, Time-Dependent Density-Functional Theory: Concepts and Applications (Oxford University Press, New York, 2011).
[22] M. A. Marques, N. T. Maitra, F. Nogueira, E. Gross, and A. Rubio, in Lecture Notes in Physics (Springer Verlag, Berlin, 2012), Vol. 837.

[23] P. E. Blöchl, Phys. Rev. B 50, 17953 (1994).

[24] G. Kresse and D. Joubert, Phys. Rev. B 59, 1758 (1999).

[25] G. Kresse and J. Furthmüller, Phys. Rev. B 54, 11169 (1996).

[26] G. Kresse and J. Furthmüller, Comput. Mater. Sci. 6, 15 (1996).

[27] See Supplemental Material at http://link.aps.org/ supplemental/10.1103/PhysRevLett.116.115004 for TDDFT implementation details and information concerning configurational averaging and the satisfaction of sum rules, which includes Refs. [28-37].

[28] X. Qian, J. Li, X. Lin, and S. Yip, Phys. Rev. B 73, 035408 (2006).

[29] A. Ojanperä, V. Havu, L. Lehtovaara, and M. Puska, J. Chem. Phys. 136, 144103 (2012).

[30] Y. Saad, Iterative Methods for Sparse Linear Systems (Siam, 2003).

[31] G. D. Mahan, Many-Particle Physics (Springer Science \& Business Media, New York, 2000).

[32] H.-C. Weissker, J. Serrano, S. Huotari, E. Luppi, M. Cazzaniga, F. Bruneval, F. Sottile, G. Monaco, V. Olevano, and L. Reining, Phys. Rev. B 81, 085104 (2010).

[33] R. T. Brown, Phys. Rev. A 2, 614 (1970).

[34] J. P. Perdew and Y. Wang, Phys. Rev. B 46, 12947 (1992).

[35] P. Gori-Giorgi, F. Sacchetti, and G. B. Bachelet, Phys. Rev. B 61, 7353 (2000).

[36] D. M. Ceperley and B. J. Alder, Phys. Rev. Lett. 45, 566 (1980).

[37] G. Ortiz, M. Harris, and P. Ballone, Phys. Rev. Lett. 82, 5317 (1999).

[38] X. Andrade, J. Alberdi-Rodriguez, D. A. Strubbe, M. J. T. Oliveira, F. Nogueira, A. Castro, J. Muguerza, A. Arruabarrena, S. G. Louie, A. Aspuru-Guzik et al., J. Phys. Condens. Matter 24, 233202 (2012).

[39] A. Schleife, E. W. Draeger, V. M. Anisimov, A. A. Correa, and Y. Kanai, Comput. Sci. Eng. 16, 54 (2014).

[40] N. Mermin, Phys. Rev. 137, A1441 (1965).

[41] G. Giuliani and G. Vignale, Quantum Theory of the Electron Liquid (Cambridge University Press, Cambridge, England, 2005).

[42] A. Pribram-Jones, P. E. Grabowski, and K. Burke, arXiv: 1509.03071.

[43] A. Sakko, A. Rubio, M. Hakala, and K. Hämäläinen, J. Chem. Phys. 133, 174111 (2010).

[44] K.-U. Plagemann, P. Sperling, R. Thiele, M. P. Desjarlais, C. Fortmann, T. Döppner, H. J. Lee, S. H. Glenzer, and R. Redmer, New J. Phys. 14, 055020 (2012).

[45] W. R. Johnson, J. Nilsen, and K. T. Cheng, Phys. Rev. E 86, 036410 (2012).

[46] M. Cazzaniga, H.-C. Weissker, S. Huotari, T. Pylkk, P. Salvestrini, G. Onida, and L. Reining, Phys. Rev. B 84, 075109 (2011).

[47] B. A. Mattern, G. T. Seidler, J. J. Kas, J. I. Pacold, and J. J. Rehr, Phys. Rev. B 85, 115135 (2012).

[48] T.-C. Li and P.-Q. Tong, Phys. Rev. A 31, 1950 (1985).

[49] T. Li and Y. Li, Phys. Rev. A 31, 3970 (1985). 
[50] E. W. Brown, J. L. DuBois, M. Holzmann, and D. M. Ceperley, Phys. Rev. B 88, 081102 (2013).

[51] V. V. Karasiev, T. Sjostrom, J. Dufty, and S. B. Trickey, Phys. Rev. Lett. 112, 076403 (2014).

[52] A. Pribram-Jones, Z.-H. Yang, J. R. Trail, K. Burke, R. J. Needs, and C. A. Ullrich, J. Chem. Phys. 140, 18A541 (2014).
[53] A. Pribram-Jones, S. Pittalis, E. K. U. Gross, and K. Burke, in Frontiers and Challenges in Warm Dense Matter, Lecture Notes in Computational Science and Engineering, Vol. 96, edited by F. Graziani, M. P. Desjarlais, R. Redmer, and S. B. Trickey (Springer Intl. Publishing, New York, 2014), p. 25 . 